\title{
Longer duration of continuous-flow ventricular assist device support predicts greater hemodynamic compromise after return of pulsatility
}

\author{
Allen S. Stewart, MD, Mark J. Russo, MD, MS, Timothy P. Martens, MD, Tariq M. Naseem, MD, Mario C. Deng, MD, \\ Ray Wang, BS, Michael Argenziano, MD, Mehmet Oz, MD, and Yoshifumi Naka, MD, PhD, New York, NY
}

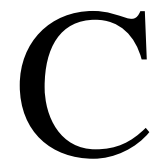
ontinuous-flow ventricular assist devices (CVADs) are more compact, less costly, and potentially more durable than pulsatile ventricular assist devices (PVADs). However, studies in animal models suggest that long-term continuous-flow support can result in hemodynamic dysfunction. This includes the disruption of the renin-angiotensin system ${ }^{1}$ and the response to norepinephrine administration in comparison with PVADs. ${ }^{2}$ The purpose of this study was to determine whether CVAD insertion and duration of CVAD support was associated with greater hemodynamic compromise after return to pulsatility after device explantation and heart transplantation.

\section{Clinical Summary}

We retrospectively reviewed 44 consecutive patients undergoing heart transplantation/ventricular assist device (VAD) explantation at our institution. These included 33 PVADs and 11 CVADs. All patients in the study had New York Heart Association stage IV congestive heart failure at the time of LVAD insertion and were successfully bridged to transplantation with no mortality on the device. Doses of all continuously infused pressors administered to patients for 7 postoperative days were collected. The degree of pressors and inotropic support was assessed by using a composite pressor/inotropic score (Table 1) that accounted for all concurrently administered vasoactive medications. To test our hypothesis that degree of hemodynamic dysfunction was directly related to duration of CVAD support, the CVAD and PVAD groups were further subdivided by using median duration of CVAD support (94 days): CVAD support for 94 days or longer (group 1, $\mathrm{n}=6$ ); CVAD support for less than 94 days (group 2, n $=5$ ); PVAD support for 94 days or longer (group 3, $\mathrm{n}=11$ ); and PVAD support for less than 94 days (group $4, \mathrm{n}=22$ ).

All patients were comparable in their preoperative characteristics with no significant differences, including age (group 1, $56 \pm 7.3$ years; group 2, $40 \pm 10$ years; group 3, $49 \pm 13.6$ years; and group $4,53 \pm 11$ years; $P=.07$ ), preoperative incidence of diabetes, hypertension, and hepatic and renal functions. Mean duration of donor heart

From the College of Physicians and Surgeons, Columbia University, New York, NY

Received for publication Aug 10, 2007; revisions received Nov 9, 2007; accepted for publication Dec 27, 2007.

Address for reprints: Mark J. Russo, MD, MS, Division of Cardiothoracic Surgery, Department of Surgery, College of Physicians and Surgeons, Columbia University, Milstein Hospital Bldg, Room 7-435, New York, NY 10032 (E-mail: mr2143@columbia.edu).

J Thorac Cardiovasc Surg 2008;136:524-5

$0022-5223 / \$ 34.00$

Copyright $(\odot 2008$ by The American Association for Thoracic Surgery doi:10.1016/j.jtcvs.2007.12.028 ischemic times was as follows: group 1, $202 \pm 40.5$ minutes; group 2, $241 \pm 27$ minutes; group 3, $185 \pm 41$ minutes; and group 4, $190 \pm 45$ minutes $(P=.2)$. During the 7-day period after heart transplantation/ VAD explantation, patients supported by CVADs had significantly higher composite scores at all time points compared with patients supported by PVADs. Among CVAD recipients, there was a direct and statistically significant relationship between duration of VAD support and composite score at all time points. Compared with patients on CVAD support for less than 94 days (group 2), PVAD support for 94 days or longer (group 3), and PVAD support for less than 94 days (group 4), patients with CVAD support for 94 days or longer (group 1) had significantly higher composite scores during the 7day period after heart transplantation (Figure 1). Nevertheless, there was no statistical difference in 30-day posttransplantation mortality between the groups. All patients receiving CVADs survived for 30 days after transplantation, whereas only 2 patients died in the PVAD group.

\section{Discussion}

These findings suggest that longer duration of CVAD support is associated with greater hemodynamic compromise, as demonstrated by the need for higher dose requirements and increased duration of pressor support after restoration of pulsatility.

This study is consistent with findings from previous studies that suggest changes in vascular tone and structure associated with periods of nonpulsatile flow. Studies in animal models demonstrate that nonpulsatile flow is associated with alternations in vascular tone. Systemic vascular resistance has also been shown to decrease significantly in nonpulsatile flow, despite pressor administration. Nishimura and colleagues ${ }^{2}$ reported a more pronounced decrease in vascular resistance in a large-animal model after 4 weeks of nonpulsatile support compared with that seen in animals treated with 2 weeks of nonpulsatile support. Similarly, continuous circulatory support has been shown to be associated with histologic changes in myocardial and endothelial tissue. ${ }^{3}$ Westaby and associates ${ }^{4}$ recently reported a significant decrease in arterial medial wall thickness after prolonged support with continuous flow devices, an effect that was associated with a decrease in smooth muscle cells and medial layer elastin content.

This is a preliminary study and has a number of limitations. First, it was retrospective in nature, and therefore the patient population might differ. Because continuous-flow devices are limited to patients enrolled in clinical trials, it is in fact likely that the patients receiving CVADs were less severely ill than those receiving PVADs; however, a comparison of baseline characteristics did not reveal any statistically significant differences between the groups. Furthermore, the composite pressor score was developed by another group of investigators for application in a non-VAD/nontransplant heart failure population ${ }^{5}$; this score has yet to be validated. 
TABLE 1. Composite pressor score

\begin{tabular}{lcccc}
\hline \multicolumn{1}{c}{ Medication } & 0 points & 1 point & 2 points & 3 points \\
\hline Milrinone $(\mathrm{mcg} / \mathrm{kg} / \mathrm{min})$ & 0.0 & $<0.375$ & $0.375-5$ & $\geq 0.5$ \\
Norepinephrine & 0.0 & $<5$ & $5-10$ & $>10$ \\
$\quad(\mathrm{mcg} / \mathrm{kg} / \mathrm{min})$ & & & & \\
Epinephrine $(\mathrm{mcg} / \mathrm{kg} / \mathrm{min})$ & 0.0 & $<0.1$ & $0.1-0.4$ & $\geq 0.4$ \\
Dopamine $(\mathrm{mcg} / \mathrm{kg} / \mathrm{min})$ & 0.0 & $<4$ & $4-10$ & $>10$ \\
Dobutamine $(\mathrm{mcg} / \mathrm{kg} / \mathrm{min})$ & 0.0 & $<5$ & $5-10$ & $>10$ \\
Phenylephrine $(\mathrm{mcg} / \mathrm{kg} / \mathrm{min})$ & 0.0 & 0.2 & 0.3 & 0.4 \\
Vasopressin (U) & 0.0 & $<2$ & $2-4$ & $>4$ \\
\hline
\end{tabular}

Further studies are needed to investigate these findings. This includes repeating the analysis on other, larger patient populations; examination of other hemodynamic measures, including systemic vascular resistance, myocardial oxygen consumption, and cardiac index; the use of gene array and serum enzyme-linked immunosorbent assay techniques to identify changes in gene expression patterns during and after periods of VAD support; and examination of changes of vascular structure by using techniques such as radial artery biopsy during and after periods of CVAD support.

\section{References}

1. Jett GK. Physiology of nonpulsatile circulation: acute versus chronic support. ASAIO J. 1999;45:119-22.

2. Nishimura T, Tatsumi E, Nishinak T, et al. Diminished vasoconstrictive function caused by long-term nonpulsatile left heart bypass. ArtifOrgans. 1999;23:722-6.

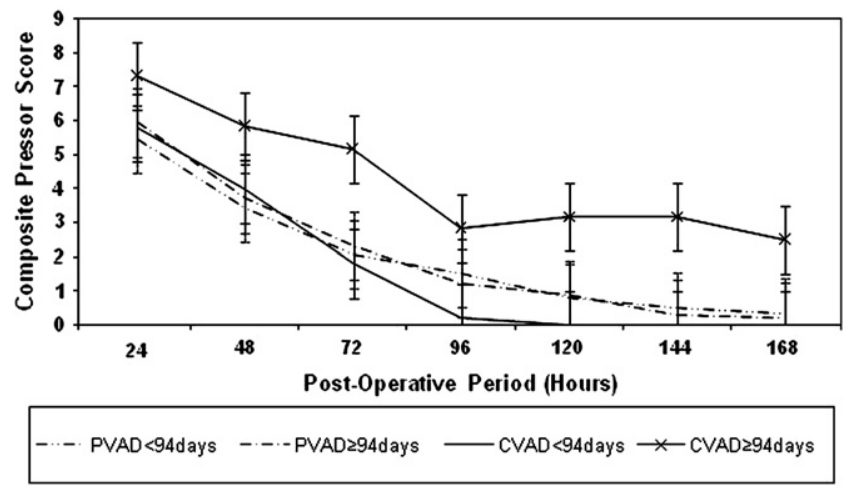

Figure 1. Composite pressor score by ventricular assist device duration and type. PVAD, Pulsatile ventricular assist device; CVAD, continuous-flow ventricular assist device.

3. Thohan V, Stetson SJ, Nagueh SF, et al. Cellular and hemodynamic responses of failing myocardium to continuous flow mechanical circulatory support using the DeBakey-Noon left ventricular assist device: a comparative analysis with pulsatile-type devices. J Heart Lung Transplant. 2005; 24:566-75.

4. Westaby S, Bertoni GB, Clelland C, et al. Circulatory support with attenuated pulse pressures alters human aortic wall morphology. J Thorac Cardiovasc Surg. 2007;133:575-6.

5. Mentzer RM Jr., Oz MC, Sladen RN, et al. Effects of perioperative nesiritide in patients with left ventricular dysfunction undergoing cardiac surgery: the NAPA Trial. J Am Coll Cardiol. 2007;49:716-26.

\section{Superficial femoral vein as substitute for pulmonary artery reconstruction after resection for bronchovascular fistula}

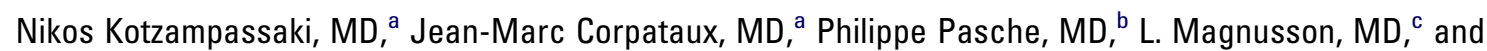

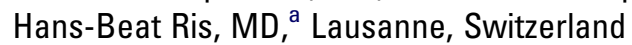

From the Divisions of Thoracic and Vascular Surgery, ${ }^{a}$ Oto-Rhino-Laryngology, ${ }^{\mathrm{b}}$ and Anesthesiology, ${ }^{\mathrm{c}}$ University Hospital of Lausanne, Switzerland.

Received for publication Aug 15, 2007; accepted for publication Oct 2, 2007.

Address for reprints: Hans-Beat Ris, MD, Centre Hospitalier Universitaire Vaudois, Service de Chirurgie Thoracique et Vasculaire, Rue du Bugnon 46, 1011 Lausanne, Switzerland.

J Thorac Cardiovasc Surg 2008;136:525-7

$0022-5223 / \$ 34.00$

Copyright $@ 2008$ by The American Association for Thoracic Surgery doi:10.1016/j.jtcvs.2007.10.071

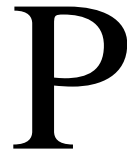

ost-lobectomy bronchovascular fistula (BVF) associated with massive hemoptysis is a rare but life-threatening complication. Surgical options include completion pneumonectomy or BVF resection with end-to-end anastomosis of the airways and reconstruction of the pulmonary artery (PA) by interposition of an appropriate substitute. We report PA resection and successful reconstruction by interposition of an autologous reversed superficial femoral vein (SFV) segment for this purpose.

\section{Clinical Summary}

A 59-year-old man with a history of coronary stenting for coronary artery disease underwent uncomplicated intrapericardial left upper lobectomy after radiochemotherapy (60 Gy) for non-small cell lung cancer. Two months later, cataclysmal hemoptysis developed in the patient, requiring cardiopulmonary reanimation, bedside rigid 